doctor teaching and discussed in clinical governance breakfast meeting.

Measurement of improvement Pre intervention audit, 30 blood tests requested in 1 week, 11 unsuccessful, pre-intervention notification $27 \%$ There was a re-audit 2 weeks after intervention which showed 46 total blood requests in 1 week, 21 unsuccessful, post intervention notification 44\% showing an improvement. A further re-audit done 1 year later showed 25 total blood tests requested in 1 week, 6 unsuccessful, 100\% notification which confirmed sustained change in practice. The same methods were used in data collection as had been in the original data collection.

Effects of changes This change created a more effective way for phlebotomists to communicate with the relevant medical team through the nurse in charge which would not compromise their time to do their job. It also allowed the medical team to act in a timely manner in the knowledge of an unsuccessful blood tests to decide how to act. It improved working relations with the doctors and phlebotomists. This change completely resolved the problem which triggered our original work and has allowed us to try to seek further ways in which the service can be more efficient.

Lessons learnt This work has taught me the importance of learning first what process is in place before setting about to make changes. I feel this project was successful because we first looked at what the phlebotomists did in their role. We created process maps to understand how the current process was working. Next time I would consider involving other wards.

Message for others Change can happen and be sustained as has been seen with our re-audit. This has meant that patient care has improved and we have minimised one of the causes of delays to decisions about patient care.

\section{G551(P) INTRODUCING A PAEDIATRIC MENTORSHIP SCHEME FOR TRAINEES IN THE SOUTH WEST REGION}

${ }^{1}$ PT van Hensbergen, ${ }^{2} \mathrm{JC}$ Sconce. 'Paediatric Department, Taunton and Somerset NHS Trust, Taunton, UK; ${ }^{2}$ Paediatric Department, Swindon and Marlborough Hospitals NHS Trust, Swindon, UK

\subsection{6/archdischild-2015-308599.501}

Context We developed a mentorship scheme for new paediatric doctors (Specialty Training Level 1) in our deanery. Mentoring is "a process of helping, supporting and encouraging paediatricians to develop their skills and to maximise their potential performance". 1 The deanery is responsible for training 150 paediatric doctors across seven hospital trusts.

Specific problem Maintaining focus on a paediatric trainee's individual career interests can be challenging in light of higher clinical workload, staffing shortages and increasing requirement for continuous standardised assessments.

Assessment of problem Paediatric services require large-scale transitions in the context of increasing workload pressures coupled to huge economic limitation. ${ }^{2}$ It is essential the workforce approaches these changes in a positive, motivated way.

Mentoring is well-established throughout different industries and organisations. It is a cost-effective, powerful personal development tool that encourages, supports and guides trainees in their individual goals. We anticipate this will benefit all parties:
- Junior trainees can readily access a friendly, reliable and supportive mentor with good working knowledge of the deanery, helpful contacts and experience of taking projects forward

- Senior trainees will improve mentoring, coaching and supervisory skills through a formal training course and develop these through practical application

- Patients and colleagues will benefit from motivated and enthusiastic trainees whose own educational and training needs are being considered

A recent study from the London Paediatric Deanery ${ }^{3}$ highlighted "a high demand for peer mentoring in paediatrics". Their programme was highly valued by participants, who gained "significant benefits including acquisition of transferable skills and positive changes in behaviour".

Intervention We arranged a mentor training day for senior trainees from the region (ST4-ST8). A full-day workshop was organised, and fully funded, by the Southwest Leadership Academy. Twelve trainees wished to provide mentoring and were able to attend the course, many others registered their interest for future workshops. Delivered content included an introduction to the mentor role, approaches to effective mentoring, mentoring models, and contracting a mentor relationship.

An interactive session followed to discuss how the mentoring programme should be developed. We produced a mentor handbook with further information on the mentor role and detail regarding the programme.

We received excellent written feedback from the day (all trainees would recommend the workshop to colleagues).

Programme design Trainees who successfully completed the mentor workshop were asked to write a 250 -word statement about themselves (11/12 completed, one withdrew for maternity leave). We did not provide further guidance on statement structure or content as we wished to promote individuality and personality.

We delivered a presentation at the ST1 induction day to advertise the scheme. We received very positive feedback, all ST1 trainees $(14 / 14)$ wished to participate.

We asked mentees to review all statements and rank them in order of preference. We were able to match all mentees to one of their "top-three" preferred mentors.

We have arranged an evening for all participants to meet together. Following this, mentor-mentee pairs will meet 2-3 monthly. We anticipate that mentor relationships will continue throughout the ST1-3 period.

Evaluation Feedback will be collected quarterly to evaluate the programme and guide future development. We aim to establish a successful rolling programme that offers effective mentoring to all future ST1 trainees starting in the deanery.

Acknowledgements We benefitted from the experiences of Sarah Eisen and Seema Sukhani, who developed a mentor scheme in the London Deanery. We are grateful for their support and guidance.

\section{REFERENCES}

1 Mentoring and clinical supervision for paediatricians. Paediatrician's Handbook, Chapter 4.6, RCPCH, 2013

$2 \mathrm{RCPCH}$. Facing the future $-\mathrm{A}$ review of paediatric services. April 2011

3 Eisen S, Sukhani S, Brightwell A, Stoneham S, Long A. Peer mentoring: evaluation of a novel programme in paediatrics. Arch Dis Child 2014;99:142-146, doi:10.1136/archdischild-2013-304277 
G552(P) CHASING OUTSTANDING INVESTIGATIONS FOR PATIENTS DISCHARGED FROM THE PAEDIATRIC UNIT: SYSTEM CHANGE

MJ Miah, GP Sinha, D Ferdinand. Paediatric Department, Manor Hospital, Walsall Healthcare NHS Trust, Walsall, UK

\subsection{6/archdischild-2015-308599.502}

Context This project was undertaken in the paediatric department of a district general hospital. We involved medical, nursing and administrative staff.

Problem There were two previous systems for chasing outstanding results for discharged patients. Those for patients seen on the Paediatric Assessment Unit (PAU) were recorded at time of discharge in a folder. Those for discharged inpatients were added to the inpatient job list with allocation to a named individual.

We found these systems resulted in investigations being seen much later than date available with the prospect of adverse impact on patient care.

Assessment of problem and analysis of its causes Random selection and analysis of 50 entries from the old system showed that $31(62 \%)$ of results had been chased/actioned. Only $9(18 \%)$ had documentation indicating results had been seen/actioned within $24 \mathrm{~h}$ of availability.

Multi-disciplinary team (MDT) discussion concluded that a major contributory factor was that investigations were being listed in the order of generation without consideration of the potential result availability date. Consequently, if a colleague chased results which were not available within their time (typically one week) on shift, these investigations would not be routinely chased resulting in potential for numerous delayed results.

Our aim was to devise a single efficient system to address all of these problems.

Intervention Following further MDT discussion a jobs book was designed. This comprised of a page-per-day diary. Any patients discharged from PAU with outstanding results were entered on the page of the anticipated result date. Those discharged from the inpatient ward with results expected beyond $48 \mathrm{~h}$ would also be entered into the diary. Each entry would include: patient and clinician details (including bleep number); job details; and outcome and documentation. The responsibility of looking at the results expected on each day was that of the team on PAU. If a result was not available when expected, then a note would be added to a revised future date to ensure follow-up.

Study design Observational study to assess effect of intervention.

Strategy for change The MDT was apprised through circulation of emails and presentation at grand-rounds of the proposed new system, rationale, aims and rules of use. Feedback was sought via email and verbally. Proposed changes were agreed with key stakeholders and colleagues before implementation. A review was planned after 6 weeks of use.

Measurement of improvement We analysed a random selection of 50 entries from each system (total 100). The new system was shown to be superior with 46 (92\%) of jobs completed (previously 62\%), with 32 (64\%) of total jobs acted upon within $24 \mathrm{~h}$ of results becoming available (previously 18\%) (Figure 1).

Effects of changes There has been a significant improvement in the chasing, actioning and documentation of results, including dates and timings which improved patient care and experience.

The team has improved ownership, traceability and accountability of clinicians who have entered and completed tasks. This

\section{Comparison of old \& new system}

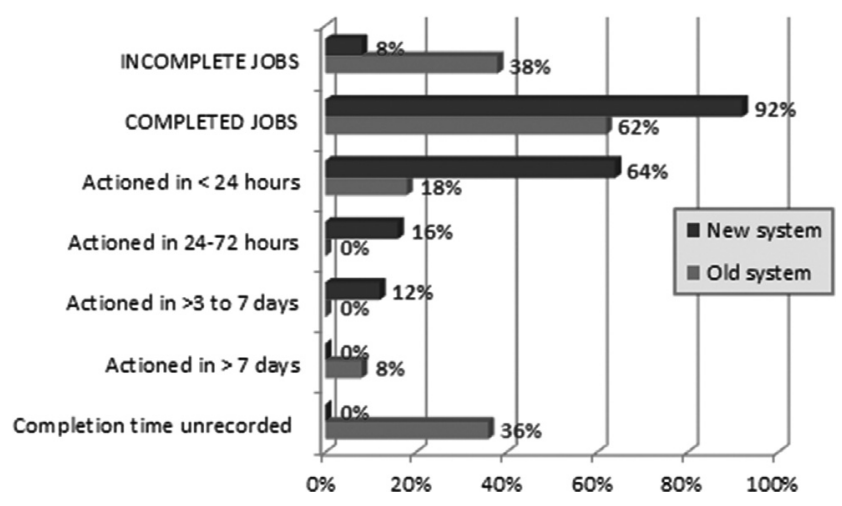

Abstract G552(P) Figure 1

encourages and reinforces a culture of teamwork to meet common goals.

The few results unavailable on the date expected are now routinely entered for chasing on an appropriate future date to ensure completion.

The new system acts as a record for future reference, audit or monitoring.

Lessons learnt Liaising with a wide variety of MDT colleagues of all grades and utilising a variety of appropriate communication methods were essential for successful system change.

Message for others We have proven that a relatively simple change in working can significantly improve patient care and foster good team working.

This system is readily replicable and can be implemented in any department to yield similar results.

\section{G553(P) RESUSCITATING RESUS}

C Edmondson, M James, S Bangalore, B Williams. Paediatrics, Northwick Park Hospital, London, UK

\subsection{6/archdischild-2015-308599.503}

Context This project was based in a busy district general's Paediatric resuscitation bay. Doctors, nurses and the resuscitation team were involved.

Problem The Resuscitation Council states: 'Healthcare organisations have an obligation to provide a high-quality resuscitation service' and 'staff have immediate access to appropriate resuscitation equipment and drugs' with 'A reliable system of equipment checks and replacement in place'. It states that appropriate airway equipment should be immediately available and circulation equipment accessible within minutes.

The current Paediatric resuscitation bay was felt to not fully meet the above criteria. The resus bay was not an intuitive area and had no clear restocking guidelines with trust incident forms being logged for missing equipment during resuscitations.

Assessment of problem and analysis of its causes Two lists of equipment were devised: one of simple airway equipment and one of equipment required to gain IV access and give a dextrose bolus. We timed one SHO trying to find specific equipment in our current resuscitation bay. The results were discussed in departmental meeting to consolidate the opinion of the Paediatric team, the Anaesthetic team, the Resuscitation officers and the Paediatric nurses, an action plan was devised to address the failings noted by staff in the department. 